\title{
Congestion Control Through EDA with Shortest Path
}

\author{
Seyed Amin Ahmadi Olounabadi, Avula Damodaram, V Kamakshi Prasad, PVS Srinivas
}

\begin{abstract}
Ad hoc mobile networks contain remote nodes linking through electronic media, without any set backend facilities. Disturbance happens in any type of intermediate nodes in these networks when data packages travel from resource to destination, leading to high package loss and also lengthy delay, triggering network efficiency destruction. This paper presents EDAODV congestion and also command directing protocol for mobile ad-hoc networks. Via determining line position and also identifying congestion degrees, EDAODV senses node-level blockage. Based on blockage rates, EDAODV makes use of the uncongested precursor and successor nodes of an overloaded node as well as starts bi-directional processes to determine alternative, uncongested courses in between them for data transmission. The algorithm discovers a lot more non-congested remedies, picking the very best solitary course for data transmission.
\end{abstract}

\section{Keywords : Congestion, EDA, EDAODV, Mobile Networks}

\section{INTRODUCTION}

Ad-hoc cordless networks are normally specified as an independent node system attached by multi-hop cordless web links [1] Among the basic jobs that an impromptu network ought to execute blockage control is to restrict the hold-up as well as barrier overflow brought on by network blockage as well as supply much better network efficiency. In wireline networks, blockage monitoring is used at the transmission degree and also is typically developed individually from various other user interface features [7] These outcomes do not use straight to cordless networks, nevertheless, as impromptu networks lead to massive package loss, high hold-up, unreasonable situations as well as reduced throughputs as each mobile node has actually restricted capability and also barrier [2] Transmitting methods can be split right into aggressive, hybrid and also responsive procedures in adhoc networks, relying on directing geography. The existing transmitting methods do not have blockage control strategies. Our inspiration is that blockage

Revised Manuscript Received on December 30, 2019.

* Correspondence Author

Seyed Amin Ahmadi Olounabadi *, Department of Computer Science and Engineering, Jawaharlal Nehru Technological University Hyderabad (JNTUH), Hyderabad, Telangana.

Avula. Damodaram, Department of Computer Science and Engineering, Jawaharlal Nehru Technological University Hyderabad (JNTUH), Hyderabad, Telangana.

V Kamakshi Prasad, Department of Computer Science and Engineering, Jawaharlal Nehru Technological University Hyderabad (JNTUH), Hyderabad, Telangana.

PVS SRINIVAS, Department of Computer Science and Engineering, Sreenidhi Institute of Science and Technology, Hyderabad, Telangana

(C) The Authors. Published by Blue Eyes Intelligence Engineering and Sciences Publication (BEIESP). This is an open access article under the CC BY-NC-ND license (http://creativecommons.org/licenses/by-nc-nd/4.0/) triggers package loss in MANETs. A brand-new viewpoint could be recognizing blockage control in the MAC or network layer. 8] Wireless is a brand-new modern technology allowing individuals to gain access to info as well as solutions no matter area. Individuals can utilize and also browse the Internet with computer systems (e.g. laptop computer, smart device as well as palmtop) whenever feasible. Wireless network can usually be split right into 2 kinds: network framework and also ad-hoc network. Mobile impromptu network (MANET) is a self-governing team of mobile customers that interact with bandwidth-limited cordless web links. Considering that hosts are mobile, network geography can alter swiftly and also unexpectedly gradually [1] Among the standard tasks for an impromptu network are website traffic administration. The primary goal of blockage monitoring is to lower network blockage latency and also barrier overflow and also give much better network efficiency [2] For wireline networks, blockage administration is used at the transmission degree as well as is commonly developed individually from various other layer functions. This strategy is rock-solid and also completely examined [wired web links have actually specified capabilities and also are self-governing 2] These outcomes do not use straight to impromptu networks, nonetheless, as impromptu networks lead to huge quantities of package loss, high hold-up, reduced throughputs as well as unreasonable circumstances. The mobile node has marginal transferring ability and also barrier in ad-hoc networks, as well as mainly intercommunicate the multi-hop relay [2] For ad-hoc networks, MANET transmitting procedures came under 3 groups based upon transmitting data upgrading system [3,4]: useful, receptive (or on-demand) and also crossbreed. Positive transmitting procedures, such as OLSR [1] and also DSDV [4] [5], effort to maintain directing details current and also constant from each node to any other node in the network. On-demand transmitting procedures such as AODV $[2,6]$ and also DSR $[8,10,11]$ When required, are just found. Crossbreed directing methods [2,10] integrate on-demand and also positive protocol attributes. For hybrid transmitting procedures, utilizing the positive technique, each node keeps transmitting info regarding its area. It makes use of non-zone on-demand transmitting method. When the network is scaled up to high node adaptability, routine transmitting data modifications due to busted links that can lead to big overhanging directing power. Classifying directing procedures has an additional measurement: congestion-adaptive directing vs congestion-adaptive transmitting. The existing transmitting procedures were congestion-un-adaptive [12-- 14] When developing a brand-new course, this remains the very same till versatility or mistake disconnects. 
Blockage can take place throughout package transmission in between resource as well as location, not covered by well-known directing protocol. It might additionally create the adhering to problems: I long hold-up, (ii) lots of package losses, and also (iii) reduced throughput. The above troubles end up being a lot more evident in massive transmission of traffic-intensive web content such as potentially multimedia details, as well as the adverse effect of package failing on solution top quality is extra considerable [2] Unlike reputable networks like the Internet, recuperation from blockage is expensive in an intricate network like a MANET [2]

Our inspiration is to get rid of that blockage is a leading source of MANET package loss. Decreasing package loss usually includes blockage control operating on top of a flexible directing protocol on the network layer [2,9] A brand-new viewpoint could be recognizing blockage monitoring in the MAC or network layer. Taking on the trouble from where it emerges can make feeling. Exceedingly high network lots is a concern very closely pertaining to tool accessibility as well as package forwarding [2,9]

Blockage is a widespread description for impromptu package goes down [15] Lu et alia [15] discovered AODV inadequate in demanding network website traffic circumstances. They recommended a changed variation of AODV (called CADV), which prefers nodes with brief queuing hold-ups in contributing to location path. While this adjustment might boost course top quality, the lengthy hold-up and also high above problems continue to be unsettled when a brand-new path requires to be uncovered. CADV is not receptive to sound. As soon as deeply crowded a well-known roadway, this offers little remedy. A load-aware transmitting protocol (DLAR) was presented in [16] The difference is that a little directing tons node is selected to be consisted of in the transmitting instructions throughout the path exploration stage.

\section{EARLY CONGESTION DETECTION TECHNIQUE}

Network blockage can happen at any kind of period, when the variety of packages going into a node surpasses its barrier capability, the node comes to be overloaded and also sheds packages. We can make use of a selection of node metrics to keep track of blockage condition. For absence of barrier room and also ordinary line up size, we can be based on the percent of all packages thrown out. We utilize very early node blockage discovery strategy to spot blockage well ahead of time. A very early blockage discovery strategy is a line up monitoring formula with arbitrary very early discovery (RED) version optimization that makes use of straight blockage standing well beforehand in a network [10].

\section{BIDIRECTIONAL PATH DISCOVERY}

The resource finds the course to the location; it transfers a RREQ package to the location, the location reacts to the initial RREQ and also returns a RREP package. RREP will certainly take a trip back in the formerly taken a trip RREQ course, including a brand-new access to its transmitting table. This roadway comes to be the key path from beginning to location. -node has 2 directing tables, a primary table (PRT) as well as a detour table (ART). PRT guides packages on the main course, while ART guides packages on alternative routes. , if ART= 0 for a node not being on any type of link's alternate course [ 17,19] A node's main path anticipates the blockage state, and also frequently produces a blockage condition package (CSP) with $\mathrm{TTL}=1$. The CSP package includes the node's blockage condition as well as a collection of criteria (resource S, location D, previous ZoneI node, each for a transmitting table location $[17,19]$

Fig reveals a streamlined instance. 3. Initially, a course S fi 1 fi 2 fi 3 fi 4 fi 5 fi $D$ for beginning $S$ to location D. This course is called S-D main path. Each package complies with the main course. At some point later on, node 3 discovers blockage and also sends out an advising to its community, its precursor node, as well as the follower node.

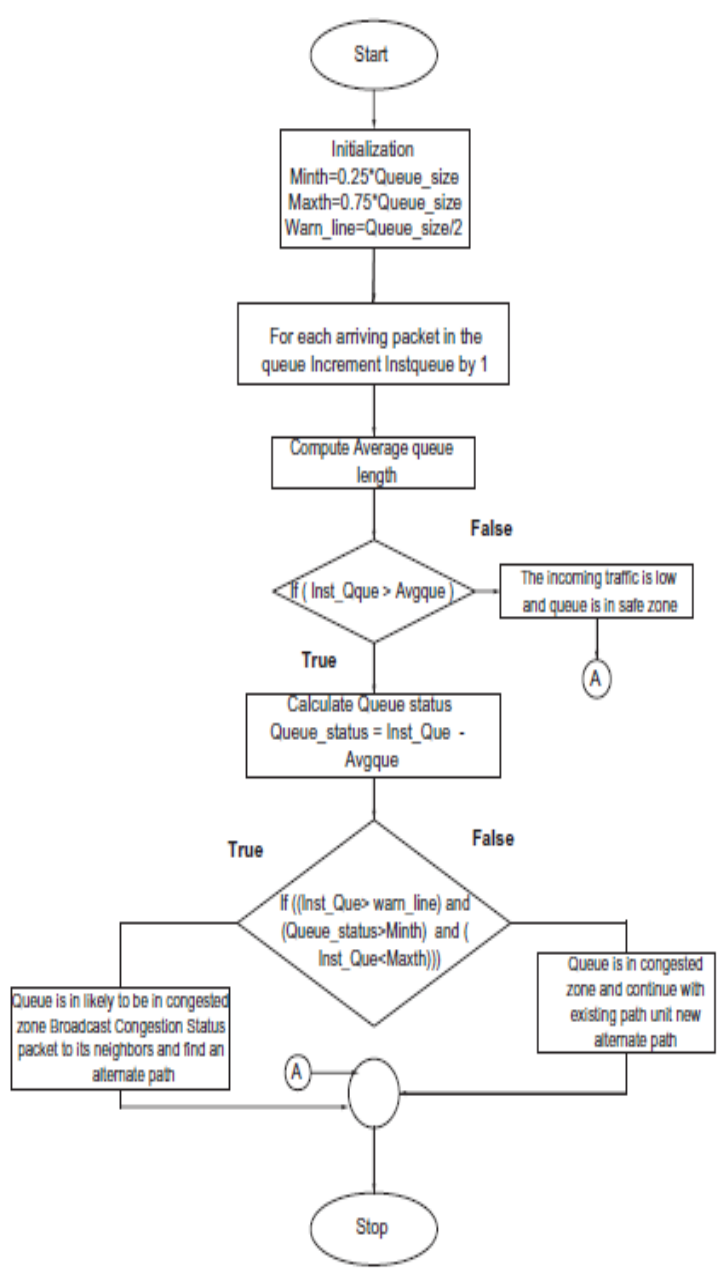

Fig: 1 Early Detection of Congestion 


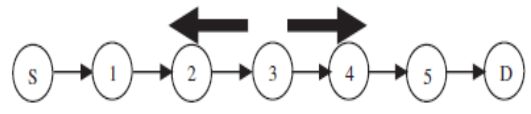

(a)

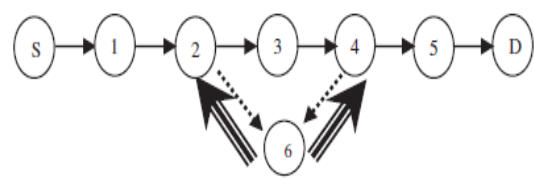

(b)

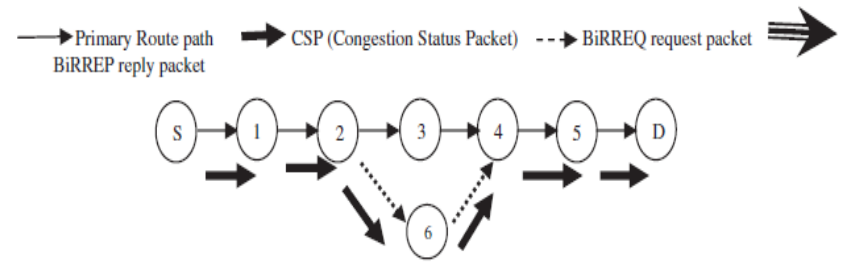

(c)

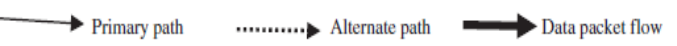

Fig: 2 EDA Path with Shortest Path

\section{NHN SET CONSTRUCTION}

This collection is chosen to cover all two-hop nodes. The NHN collection of resource host $\mathrm{S}$, signified by $\mathrm{NHN}(\mathrm{S})$, is after that an approximate part of S' non-congested one-hop area that fulfills the complying with problem: each node in S's rigorous two-hop community need to have a web link to NHN( S) and also ought to not fall under busy area. The NHN configuration is an initialization treatment, each mobile host computes its blockage standing occasionally making use of very early blockage discovery formula. Each mobile host transfers its blockage standing to its one-hop next-door neighbors on the network, utilizing a CSP (blockage condition package). Currently, each mobile host recognizes its uncongested one-hop next-door neighbor nodes as well as files the data in its uncongested one-hop data source. Afterwards, each mobile host exchanges its one-hop uncongested next-door neighbor info, learning its two-hop uncongested next-door neighbor nodes. At this phase, each mobile host develops its NHN-set by picking a part of its one-hop, non-congested bordering nodes to make sure that the sub-set mobile hosts can ahead their program web traffic to bordering two-hop nodes to decrease flooding website traffic.

\section{PERFORMANCE EVALUATION AND ITS METRICS}

In [21] includes efficiency indications EDAPR, EDCSCAODV and also EDAODV. Contrast of EDAPR's result with MANET's EDAODV as well as EDCSCAODV transmitting procedures is made. Hence the monitoring exists listed below as: We took into consideration the adhering to essential metrics:

- Packet Delivery Ratio (PDR): the proportion in between location variety of packages gotten as well as resource variety of packages sent out.

- End-to-end hold-up: a package has a hard time from shedding the s ender to the recipient.

- Overhead directing: complete variety of packages sent out throughout simulation procedure. For packages sent out over a number of jumps, every jump transmission is dealt with as one transmission.

\section{SIMULATION RESULTS}

The network contained 100 nodes of $1400 \mathrm{~m}$ terrine dimension. Radio range is $250 \mathrm{~m}, 2 \mathrm{Mbps}$ data transfer. The MAC layer utilized IEEE 802.11 DCF (dispersed interaction feature). We made use of 2ray area representation design templates to transfer streams. A MAC layer user interface line up can bring 50 packages prior to being sent out to the physical bridge. MAC-layer comments discovered web link damage. A network directing barrier can accumulate to 64 data packages. This barrier maintains data packages awaiting a course, like packages for which path exploration has actually begun however no reaction has actually yet gotten here. We utilized EDAPR, EDAODV, and also EDCSCAODV transmitting procedures. The data circulation utilized CBR differs from 4 packages to 16 packages and also moves from 10 to 50 circulations. Optimum node rate is 10 $\mathrm{m} / \mathrm{s}$ as well as simulation time is $900 \mathrm{~s}$.

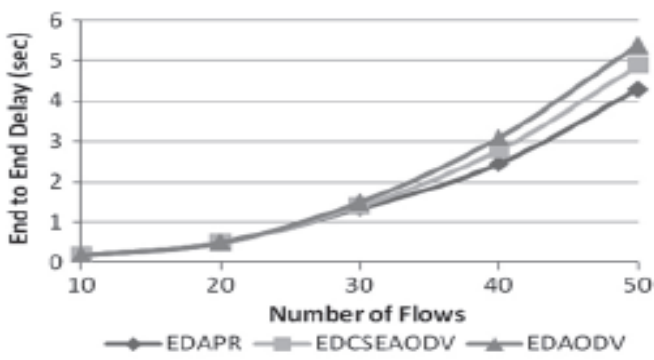

(a) End to End delay

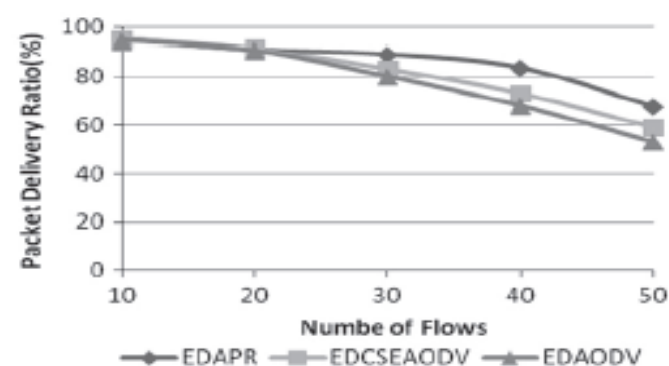

(b) Packet delivery ratio

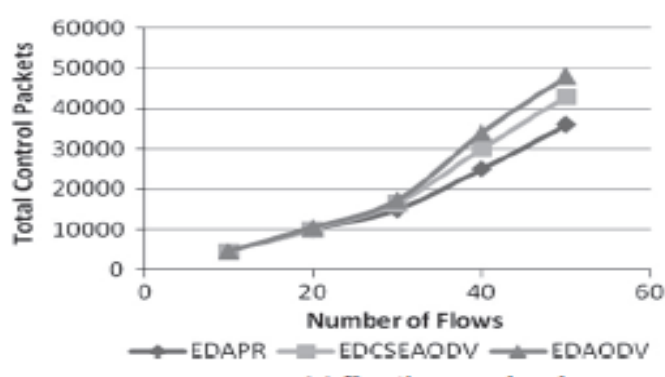

(c) Routing overhead

Fig: 3 Performance Evaluation

\section{CONCLUSION}

In this paper, we recommended a brand-new type of blockage administration within mobile multihop networks: very early blockage forecast as well as vibrant transmitting in MANET (EDAPR). EDAPR deals with less package losses than various other congestion-free methods.

Published By:

Retrieval Number: B3661129219/2019@BEIESP

DOI: 10.35940/ijeat.B3661.129219

Blue Eyes Intelligence Engineering

Journal Website: www.ijeat.org 
This is since EDAPR attempts to prevent first-place blockage, as opposed to taking care of it reactively. An essential to EDAPR style is choosing NHN nodes. The NHN node recognizes a prospective blockage in advance. This calls for a non-congested course in between resource as well as location to prevent web traffic. EDAPR does not sustain high expenses to situate uncongested paths as only NHN nodes ahead the command packages throughout floodings. The technique dramatically decreases expenses contrasted to existing flooding system. It likewise checks blockage throughout data transmission. It adjusts blockage to discover an alternate path via NHN if blockage is most likely to take place. EDAPR additionally supplied a quick end-to-end time out over various other approaches. Our ns-2-based simulation sustained the benefits of EDAPR as well as revealed lowered end-to-end latency, transmitting expenses and also boosted package circulation proportion over EDAODV as well as EDCSCAODV.

\section{REFERENCES}

1. Siva Ram Murthy C, Manoj BS. Ad hoc wireless networks architectures and protocols. Pearson Edu 2007.

2. Duc A, Tran, Harish Raghavendra. Congestion adaptive routing in mobile ad hoc networks. IEEE Trans Parallel Distrib Syst 2006;17(11):16-28.

3. Corson S, Macker J. Mobile ad hoc networking (MANET): routing protocol performance issues and evaluation considerations. RFC 1999;2501.

4. Johnson, Maltz D. Ad hoc networking. Addison-Wesley; 2001.

5. Perkins CE. Highly dynamic destination-sequenced distancevector routing (DSDV) for mobile computers. Proc ACM SIGCOMM 1994:234-344.

6. Perkins CE, Belding-Royer EM, Chakeres I. Ad hoc on demand distance vector (AODV) routing. IETF Internet Draft 2003.

7. Gui C, Mohapatra P. A framework for self-healing and optimizing routing techniques for mobile ad hoc networks. ACM Trans Wireless Networks 2008;14(1):29-46.

8. Broch J, Johnson D, Maltz D. The dynamic source routing protocol for mobile ad hoc networks. IETF Internet Drafts 1999.

9. Christian Lochert, Bjo“ rn Scheuermann, Martin Mauve. A survey on congestion control for mobile ad-hoc networks. Wiley Wireless Commun Mobile Comput 2007;7(5):655-76.

10. Floyd S, Jacobson V. Random early detection gateways for congestion avoidance. IEEE/ACM Trans Networking 1993;1(4):397-413.

11. Feng Gang, Agarwal AK, Jayaraman A, Siew Chee Kheong. Modified RED gateways under bursty traffic. IEEE Commun Lett 2004;8(5):323-5.

12. Li VOK, Lu Zhenxin. Ad hoc network routing 2004. In: IEEE international conference on networking, sensing and control, vol. 1; 2004. p. 100-5.

13. Ramanathan R, Redi J. A brief overview of ad hoc networks: challenges and directions. Commun Mag IEEE 2002;40(5):20-2.

14. Royer E, Toh CK. A review of current routing protocols for ad hoc mobile wireless networks. IEEE Pers Commun 1999;6(4):46-55.

15. Lee SJ, Gerla M. Dynamic load-aware routing in ad hoc networks. Proc IEEE Intl Conf Commun 2001:3206-10.

16. Lu Y, Wang W, Zhong Y, Bhargava B. Study of distance vector routing protocols for mobile ad hoc networks. Proc IEEE Intl Conf Pervasive Comput Commun (PerCom) 2003:187-94.

17. Senthil Kumaran T, Sankaranarayanan V. Early detection congestion and control routing in MANET. In: Proceedings of the seventh IEEE and IFIP international conference on wireless and optical communications networks (WOCN 2010); 2010. p. 1-5.

18. Senthil Kumaran T, Sankaranarayanan V. Early detection congestion and self cure routing in MANET. In: Proceedings of Springer LNCS computer and information science, vol. 142 (Pt. 3); 2011. p. 562-7.

19. Yingqun $\mathrm{Yu}$, Giannakis GB. Cross-layer congestion and contention control for wireless ad hoc networks. IEEE Trans Wireless Commun 2008;7(1):37-42.

20. Yun-Sheng Yen, Hung-Chieh Chang, Ruay-Shiung Chang, HanChieh Chao. Routing with adaptive path and limited flooding for mobile ad hoc networks. Elsevier transaction on computers and electrical engineering, vol. 36(2); 2010. p. 280-90.

\section{AUTHORS PROFILE}

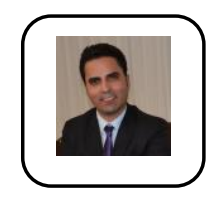

Management.

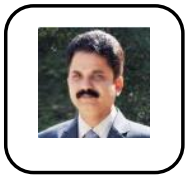
and Faculty of Computer Science \& Engineering Department, Jawaharlal Nehru Technological University Hyderabad (JNTUH), Hyderabad, (Telangana) India. Research interests: include Image Processing, Pattern Recognition, Network Security, Steganography and Digital Watermarking.

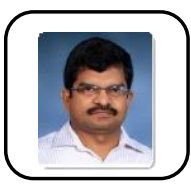

Prof. V Kamakshi Prasad, Director of DE, Professor and Faculty of Computer Science and Engineering Department, Jawaharlal Nehru Technological University Hyderabad (JNTUH), Hyderabad, Telangana, India. Research interests: Speech Recognition and Processing, Image processing, Pattern Recognition, Data Mining, Ad-hoc networks, Computer Graphics.

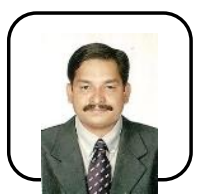

Prof. PVS SRINIVAS, Professor and Faculty of Computer Science \& Engineering, Sreenidhi Institute of Science and Technology (SNIST College) Hyderabad (Telangana) India. Research interests: Computer Networks, Cloud Computing and IoT.

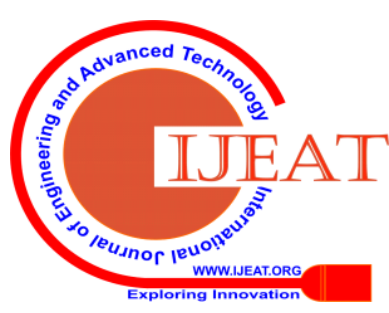

